\title{
Gobernanza metropolitana en el Reino Unido
}

\section{M ark Kleinman*}

\section{Gobernanza urbana en Gran Bretaña}

Del mismo modo que nuestro dinero, nuestra gastronomía y el lado de la carretera por el que conducimos son diferentes de la mayoría de los demás lugares de Europa, también nuestras ciudades tienen al gunas características clave que las distinguen de las ciudades de cualquier otro lugar. Estas diferencias incluyen las siguientes.

En primer lugar, el poder económico, político y cultural en el Reino Unido se halla muy centralizado en Londres. El poder está generalmente más disperso en otros países europeos, aunque existen importantes paralelismos entre Gran Bretaña/Londres y Francia/ París.

En segundo lugar, la base legal y constitucional de los gobiernos de las ciudades en el Reino Unido es muy diferente de la mayoría del resto de los países europeos. Dicha base se refiere a la combinación de un Estado unitario, una Constitución no escrita y la tradición del common law. Los gobiernos de las ciudades tienen bastante poco de autoridades constitucional mente independientes - son fundamentalmente criaturas del Parlamento- . Este hecho contrasta con la «tradición de las ciudades y barrios libres o con carta, basados en la aceptación por parte del Estado de la contribución que étas llevan a cabo a la riqueza compartida como centros libres de riqueza y comercio, que es virtualmente una raíz común en todos los sistemas europeos) (N ORTON, 1991: 24).

En tercer lugar, y en parte como resultado de los dos primeros factores, el Gobierno Local en Gran Bretaña es débil tanto desde el punto de vista político como fiscal. D entro del gobierno unitario del Reino Unido, el Gobierno Local ha sido siempre una criatura del Gobierno Central, aunque durante los Gobiernos de Thatcher y M ajor su situación se deterioró considerablemente. Sin embargo, el programa de «devolución»1 del N uevo Laborismo ha cambiado este panorama en alguna medida, con la introducción de niveles de gobierno intermedio, regional 0 «meso», con poderes variados en Escocia, G ales y Londres, y con la posibilidad de un gobierno regional inglés en el futuro.

En cuarto lugar, existen algunas diferencias clave también en el modo en que se financia el nivel subnacional de gobierno. En el Reino U nido hay, al menos en teoría, una equiparación fiscal entre áreas. M ás aún, desde la nacionalización del impuesto sobre las actividades económicas por el gobierno Thatcher en 1990, no hay ventajas fiscales directas - reales o percibidaspara que los gobiernos locales atraigan nueva inversión. Además, tanto el gasto local como la capacidad fiscal pueden potencialmente limitarse. D esde el punto de vista de las ventajas fiscales, las ganancias en el nivel local procedentes de las políticas para el desarrollo (y a la inversa, el desincentivo fiscal para ralentizar el crecimiento o las políticas anti-crecimiento) son prácticamentenulas.

En quinto lugar, el sistema electoral de $G$ ran Bretaña de «first past the post» (el primero consigue el puesto), la relativa debilidad de los niveles intermedios y el sistema de partidos del modelo Westminster, dan lugar a un estilo de política nacional peculiar en relación con el existente en muchos países europeos. El casi europeo Estado de Bienestar británico le hace distinto de los EEUU; pero su forma anglosajona de bienestar le hace diferente de Europa - diverso tanto respecto de los más extensos Estados de Bienestar de los países nórdicos como del modelo «Rhineland» de Estado de Bienestar corporatista- .

En sexto lugar, la implicación del mundo de los negocios en la política urbana y en la gobernanza es débil, comparada con las 
coaliciones por el crecimiento de los EEUU, o con las Cámaras de Comercio con papel estatutario en los países europeos.

En séptimo lugar, hay actitudes anglosajonas compartidas que aproximan, en algunos aspectos, el Reino Unido a los EEUU y, en alguna medida, a Australia y a N ueva Zelanda. Estas amplias características culturales e institucionales incluyen: escepticismo hacia el papel del Estado; tradición del common law; pragmatismo y prioridad de la libertad sobre la igualdad. Al mismo tiempo, el Reino Unido posee algunas actitudes «europeas» compartidas que incluyen: un consenso en el Estado de Bienestar; una confianza en el papel impulsor del Estado; soberanía compartida con la Unión Europea, y una posible moneda única en el futuro.

Las estructuras de la gobernanza de las ciudades del Reino Unido difieren de las de cualquier lugar en Europa, pero las autoridades hacen frente a problemas similares para alcanzar los objetivos del crecimiento económico y la cohesión social. En todos los países, la transformación de la gobernanza y la incre mentada fragmentación de la autoridad suponen un mayor énfasis en el papel de las ciudades como actores, llevando conjuntamente diferentes agencias para alcanzar los objetivos de la competitividad y de la cohesión. El sistema emergente es el de la gobernanza multinivel, con papeles cambiantes en la ciudad, la región y en los niveles nacional y europeo. Para algunos comentaristas, G ran Bretaña está retrasándose en lo que es percibido como un desarrollo universal de la gobernanza multinivel, que a menudo parece incluirse en la agenda en términos de jue go de suma cero:

«H abrá conflicto y competitividad, porque lo que es bueno para una región puede no siempre ser bueno para un vecindario concreto de dicha región... Es necesario un sistema efectivo de gobernanza multinivel para gestionar conflictos y fortalecer partenariados.. La agenda no consiste en elegir entre estas opciones, sino en encontrar espacio y extensión para todas ellas... Se puede tener mucho gobierno y no tener demasiada gobernanza» (STOKER, The Guardian, 13 de junio de 2001).

Algunas ciudades parecen ser más efectivas que otras en perseguir y lograr objetivos. El liderazgo urbano parece ser un aspecto del que las ciudades británicas carecen. Un estudio comparativo entre ciudades de Francia y de Gran Bretaña encontró importantes similitudes, pero también importantes diferencias: «el gran papel del sector privado en la financiación de los proyectos de desarrollo modifica la agenda de las políticas públicas. En las re laciones políticas de nuestras dos ciudades francesas no había un vacío como el que encontramos en Southampton y, por extensión, en Leeds.. El poder de la mayor parte de los líderes electos de Francia aseguraba la coordinación del complejo patrón de las agencias.» (JOHN Y COLE, 2000: 88).

\section{La política de las ciudades bajo los gobiernos conservadores (1979-1997)}

En Gran Bretaña, a finales de los setenta, la agenda de dere chas radical de las Administraciones Thatcher definió el gasto público como el corazón del problema económico. La solución thatcheriana fue intentar «reducir las fronteras del Estado» a través de diversas formas de privatización. En realidad, el gasto público total continuó incrementándose en términos reales durante los ochenta y los noventa (aunque más lentamente que durante los años sesenta y principios de los setenta), y el gasto público, en términos porcentuales sobre el Producto $\mathrm{N}$ acional Bruto, fue de hecho más bajo en el período de la Administración laborista después de 1997 que durante los gobiernos de Thatcher y $\mathrm{M}$ ajor.

Sin embargo, después de finales de los setenta se produjo también un marcado cambio ideológico en el que el Gobierno - particularmente el Gobierno Local, e incluso más específicamente en su forma tradicional municipal - era considerado más como el problema que como la solución. Este cambio ideológico en el corazón del Gobierno Central produjo una reacción de los laboristas en el gobierno local, particularmente en las grandes ciudades. En oposición al modelo thatcheriano, estos Ayuntamientos no ofrecieron las viejas políticas laboristas tradicionales, sino una visión de la N ueva Izquierda de socialismo local, basado en nuevas alianzas sociales, una política radical y nuevas formas de control democrático. Un cierto número de barrios de Londres y el Ayuntamiento del Gran Londres impulsaron tal enfoque más allá de sus límites exteriores.

A finales de los años ochenta era evidente que esta forma de política insurreccional institucionalizada había fracasado, pero también la Nueva D erecha poséa una visión radical del gobierno (RIDLEY,1988), de la que los Consejos locales fueron ampliamente excluidos. Las condiciones externas fueron, de este modo, una base para el desarrollo de la siguiente fase de la innovación en la gobernanza urbana, en la que el concepto clave era el partenariado - tanto entre el gobierno central y el local, como entre gobierno, empresariado y «sociedad civil»» . Basándose en la experiencia de las políticas del gobierno conservador, tales como la Estate Action y otros programas, el City Challenge, introducido a principios de los noventa, proporcionó la estructura para el nue vo enfoque.

Las autoridades locales volvieron al centro de la política urbana (en contraste con las Corporaciones para el D esarrollo U rbano de los ochenta, que se habían designado explícitamente para excluirles), aunque se les incentivó - con subvenciones- para crear partenariados genuinos con empresarios locales y representantes de la «comunidad». Sin embargo, la financiación del City Challenge se restringió a la mayoría de las áreas deprimidas y las subvenciones no se destinaron a necesidades básicas, sino al for- 
talecimiento de ofertas competitivas procedentes de los partenariados posibles. El sistema combinaba aspectos relacionados con las necesidades básicas con los propios de la localización compe titiva; contenía tanto elementos top-down (de enfoque descendente) como bottom-up (de enfoque ascendente), y fortalecía el control central sobre el gasto en rehabilitación, en relación con la descentralización. Posteriores programas y políticas desde entonces - Single Regeneration Budget (Presupuesto Ú nico para Rehabilitación), N ew Deal for Communities (N uevo Acuerdo para las Comunidades), Employment Zones (Zonas de Empleo), H ealth and Education Action Zones (Zonas de Acción en Educación y Salud) - compartían estos elementos clave, a pesar de que el equilibrio entre los diferentes aspectos ( $p$. ej. la iniciativa central 0 local, las necesidades básicas frente a la localización competitiva, la extensión de los partenariados en funcionamiento) había cambiado en el tiempo y dentro de los programas.

¿Y qué fue de las grandes ciudades, en concreto? Las soluciones a la cuestión de la gobernanza metropolitana generalmente adoptaron una de las dos formas siguientes: la intermunicipalidad y la supramunicipalidad (LefĖVRE,1998). Ésa es la solución que se puede al canzar de los ajustes (voluntarios o estatutarios) entre autoridades independientes que retienen sus competencias actuales; 0 que puede suponer la creación de un nuevo nivel te rritorial de gobierno con funciones definidas, competencias y presupuestos. Con la creación del Consejo del Gran Londres en 1965, Gran Bretaña se situó a sí misma firmemente en el polo supramunicipal. En la mayoría de los países europeos se va actualmente en la dirección contraria: el Gran Rotterdam fue disuelto en 1985 y la Corporación M etropolitana de Barcelona en 1987. La intermunicipalidad es más común: por ejemplo, la creación de cuatro Comunidades U rbanas (Communautés U rbaines) en Francia con representantes elegidos indirectamente (Burdeos, Lille, Lyon, Estrasburgo) (LEFÈVRE, 1998).

\section{7: el nuevo laborismo en el Gobierno}

Por lo tanto, la búsqueda de nuevas formas de gobernanza urbana es un proceso que se ha ido desarrollando durante más de veinte años y posee continuidades y diferencias entre las fases Tory y del nuevo laborismo, durante todo este proceso. Al igual que sus predecesores conservadores, los nuevos laboristas ven muchos Consejos locales como burocráticos, ineficientes y ofreciendo poco value for money (sentido de la eficiencia económica). H a continuado el marco básico del sistema de gobierno central-local heredado - fuerte control central, autonomía fiscal restringida, establecimiento de objetivos y medición de eficacia y el requisito para las autoridades locales de actuar en partenariado con el sector del empresariado local y con las comunidades locales-.
Pero la política del nuevo laborismo no ha sido reducir aún más el papel del Gobierno Local (como los conservadores podrían haber hecho), sino, más bien, modernizar el Gobierno Local. Las autoridades locales continúan siendo importantes para la gobernabilidad local y regional - una lección aprendida durante los años de Thatcher y M ajor- . I gualmente, sin embargo, está claro que el nuevo laborismo espera que las autoridades locales gestionen de un modo empresarial e inclusivo, y empleará los métodos del central ismo británico para asegurarse de que actúan de acuerdo con los deseos del Gobierno.

Cuando los laboristas tomaron posesión en 1997, el Partido Laborista halló al Gobierno Local en Inglaterra en un estado de depresión. Los años de reforma, que habían afectado a sus estructuras, sus funciones y sus finanzas, habían desmoralizado del mismo modo a los Consejeros y a los empleados públicos (véanse SteWART y Sto Ker, 1995). El gasto público había sido constreñido durante más de veinticinco años, con la consecuencia de que muchos Consejos estaban proveyendo servicios públicos colapsados. Los Consejeros de todos los partidos habían imaginado que un gobierno laborista entrante les liberaría de algún modo y les facilitaría más presupuesto para los servicios locales. En realidad, el Gobierno de Blair simpatizaba poco más con el Gobierno Local que sus predecesores Tory.

El Gobierno Local en Gran Bretaña ha sufrido, a largo plazo, una pérdida de autoestima y de poder (véase LOU GH LIN et al., 1985). Los servicios públicos que habían desarrollado originariamente durante el siglo XIX y principios del XX las ciudades y los ayuntamientos, habían sido separados del control local. Las infraestructuras como el gas, el agua y la electricidad habían sido nacionalizadas y después privatizadas. La Sanidad (en la que el Consejo del Condado de Londres había sido un referente (véanse G IBBON y BELL, 1939) fue ampliamente apartada del control del Consejo en 1948 y totalmente en 1974. Las ambulancias también se transfirieron al control nacional (desde el Consejo del Gran Londres) en 1974. El desarrollo de un «Estado de Bienestar» en el que los servicios eran gratuitos en el lugar de provisión y, fundamentalmente, consistentes a lo largo del país, hizo más difícil la tarea de justificar las variaciones en los servicios locales. Westminster y W hitehall intentaron imponer la más elevada uniformidad. Como resultado, cada vez más, todo aqueIlo que había sido G obierno Local pasó a convertirse directa 0 indirectamente en objeto de control central.

En sus dos primeros años de mandato, el Gobierno Blair, tal como había prometido en la campaña electoral, mantuvo los planes de gasto público de la Administración conservadora saliente. La gobernanza en $\mathrm{G}$ ran Bretaña se mantuvo básicamente centralista. Los poderes del Gobierno Local son controlados por el Parlamento; el gobierno sub-nacional no tiene autoridad constitucional independiente. El gasto del Gobierno Local se controla muy estrechamente. 
Sin embargo, el nuevo laborismo está comprometido también en mostrar una aproximación diferente en el Gobierno, no sólo distinta respecto de la de los conservadores a los que reemplazaron en su mandato, sino incluso respecto de los gobiernos del «viejo laborismo» del pasado. Las palabras clave para el Gobierno Blair eran (y siguen siendo): «modernización»; «reforma»; «mercados»; y «oportunidad». Se ha intentado modificar el viejo sistema de funcionamiento burocrático de los Consejos locales y fortalecer un enfoque más dinámico. El Gobierno ha argumentado que esta medida reforzará la democracia local. Los críticos afirman lo contrario: que las decisiones serán tomadas por un menor número de personas y que el resultado será menos democrático. M uchos miembros de los Consejos han afirmado que su papel se verá disminuido como resultado de las reformas del Nuevo Laborismo.

Según la Ley de Gobierno Local del 2000, los Consejos de ben consultar a sus comunidades y después presentar una nueva constitución. Para las grandes zonas urbanas está nueva constitución habrá de adoptar una de las tres opciones siguientes:

i) un Alcalde por elección directa con un «gabinete»,

ii) un gabinete con un líder elegido por el C onsejo,

iii) un Alcalde elegido directamente por el pueblo con un ge rente del Consejo elegido por el Consejo.

Es más, en el Libro Blanco del Gobierno Urbano, se argumenta que «la experiencia internacional sugiere que los Alcaldes por elección directa son a menudo la mejor opción para ofrecer el liderazgo que las más grandes ci udades y villas necesitan».

Un componente importante de este enfoque para el gobierno fue el programa de «devolución» del Nuevo Laborismo. En 1999, se instituyeron el Parlamento escocés y la Asamblea galesa, y en el 2000 la Autoridad del Gran Londres con su Alcalde por elección directa. El Parlamento escocés tiene poderes legislativos sobre la mayor parte de los asuntos de política interior, y una capacidad limitada (aunque compleja) en la modificación del impuesto sobre la renta. A la Asamblea de Gales se le otorgaron poderes mucho más limitados, y no tiene competencia para variar los impuestos. Los G obiernos escocés y galés y la Autoridad del Gran Londres son importantes desde un punto de vista simbólico como el inicio de centros alternativos de poder político. Después de tres o cuatro años, existen al gunas diferencias emergentes en la política de Escocia y Gales en comparación con el resto de Gran Bretaña.

M uchos diputados laboristas y simpatizantes esperaban que la «devolución» llegara más lejos, y condujese a Inglaterra a un gobierno regional. Pero la aproximación del Nuevo Laborismo en el poder fue cauta y a veces confusa. El N uevo Laborismo creó nueve Agencias de D esarrollo Regional en Inglaterra, incluida la Agencia de D esarrollo de Londres bajo la dirección del
Alcalde. Las otras ocho Agencias de D esarrollo Regional se pusieron en marcha en abril de 1999. Con la excepción de Londres, éstas son instituciones designadas, no elegidas, encargadas de coordinar el desarrollo económico regional y la reactivación, mejorar la competitividad, y reducir la pobreza en sus áreas. La financiación procede del Gobierno Central. Los presupuestos no son amplios - para cada Agencia de D esarrollo Regional varía entre treinta millones de libras y ciento setenta y seis millones- . En el 2001, el Gobierno anunció un incremento de la financiación y una mayor flexibilidad de gasto para las Agencias de De sarrollo Regional. Junto a las Agencias de Desarrollo, se han constituido Cámaras Regionales - grupos voluntarios de Consejeros, empresarios, grupos de la comunidad y sindicatos- . Estas Cámaras o A sambleas, que intentaron asegurar alguna forma de representatividad local, se constituyeron con miembros de las autoridades locales elegidos indirectamente dentro de sus áreas, más una minoría de individuos procedentes de otras organizaciones representativas. En teoría, éstas podrían evolucionar hacia Parlamentos Regionales en el futuro.

A pesar de la efectiva cesión de poder a Escocia, Gales, Irlanda del N orte y Londres, la «devolución» en Inglaterra (salvo en la capital) experimentó una reforma demasiado lejana de los planteamientos de los modernizadores constitucionales del Nuevo Laborismo. Los informes de la prensa entre 1997 y 2001 sugirieron que el gabinete permanecía dividido acerca de la cuestión. Por una parte, el D eputy Prime M inister, John Prescott, se mostró como un entusiasta del poder regional, mientras que, por otra, el Secretario de Interior, Jack Straw, era altamente escéptico. El Gobierno Local, que tenía la gran sospecha de que cualquier movimiento hacia el Gobierno Regional tendría consecuencias dañinas para los Consejos (especialmente para los Consejos de Condado), no era un entusiasta del cambio, excepto, quizás en el Nordeste.

Tony Blair nunca ha sido un defensor del Gobierno Regional. El primer gobierno Blair no hizo ningún movimiento hacia un gobierno regional plenamente desarrollado en Inglaterra. El adalid del Regionalismo en el Gabinete era claramente John Prescott, cuya influencia decreció después de la segunda victoria laborista. El interés por la política regional ha estado más fragmentado que consolidado desde junio de 2001. Es igualmente evidente que el énfasis del Primer M inistro - y por tanto de su Gobierno- se situaba en la prestación de los servicios públicos, no en nuevos cambios constitucionales.

En mayo del 2002, el Gobierno publicó un Libro Blanco para la «devolución» regional en Inglaterra que perfilaba una propuesta de regiones con pocos poderes (generalmente menos que los de la Autoridad del Gran Londres) y con independencia financiera muy limitada (DTLR, 2002). Es más, el impulso se mantenía centralista. El Gobierno Regional sólo se pondría en marcha en donde se solicitara un referéndum, e incluso sólo en- 
tonces a expensas de eliminar uno de los niveles territoriales inferiores en donde existieran dos - presumiblemente los Consejos de condado- . Los poderes de los nuevos gobiernos regionales, tal como están establecidos, procederán de los «quangos» y de las oficinas regionales del Gobierno, pero no reduciendo los poderes existentes en el Estado Central.

\section{La actual agenda de la política urbana en el Reino Unido}

La «agenda urbana» en Gran Bretaña puede quizá ser entendida mejor como operando en tres niveles.

- Primero, hay políticas urbanas en sentido estricto (y estrecho), por ejemplo, rehabilitación urbana, vivienda y planificación.

- Segundo, y más ampliamente, la configuración amplia del Gobierno Local y las políticas centrales locales, en tanto que afectan a las ciudades.

- En tercer lugar, existen al gunas grandes prioridades del Gobierno - prestación de servicios en salud, educación, reducción de la delincuencia y transporte público; y promoción de la competitividad / desarrollo sostenible-. Aunque estas políticas no sean específicamente «urbanas), suponen un gran impacto en las ciudades.

Como una parte más propiamente «urbana» de la agenda, al llegar al poder, el nuevo Laborismo puso en marcha la «U rban Task Force» (el Grupo de Trabajo U rbano), bajo la dirección del arquitecto Lord Richard Rogers, con el fin de «identificar las causas del declive urbano en Inglaterra y recomendar soluciones prácticas para atraer a la población de nuevo a nuestras villas, ciudades y vecindarios urbanos. Establecerá una nueva visión de la re habilitación urbana fundamentada en los principios del diseño de la excelencia, el bienestar social y la responsabilidad medioambiental con un marco económico y legal viable.»

La «Task Force» se presentó en junio de 1999; al año siguiente en que el Gobierno publicara el Libro Blanco Urbano, el primero en veinte años. Sin embargo, más recientemente, Richard Rogers, director de la «U rban Task Force», había perdido la paciencia respecto del Gobierno, según sus artículos de prensa (The Guardian, 26, 01, 2002):

«Yo no estoy satisfecho, pero podría expresarlo mucho más contundentemente. Si no lo hacemos ahora, nunca podremos competir con los mejores y no podremos, incluso, ni ponernos al día con Europa. La organización que debiera interpretar la visión no está ahí. No he escuchado al Primer M inistro, 0 al "Chancellor", expresar una visión para las ciudades y villas, y sin eso no hay nada que hacer».
El D epartamento deTransporte, Gobierno Local y las Regiones (desde el verano de 2002, repartido entre el $D$ epartamento para el Transporte y la 0 ficina del $D$ eputy Prime M inister) era el ministerio encargado de la tarea de llevar las ideas del Libro Blanco a la práctica. EI DTRL, en su plan de implementación para el Libro Blanco U rbano, identifica los siguientes grupos de tareas:

- Personas que comparten su futuro: programas de financiación de partenariados y vecindarios, incluyendo los Partenariados Estratégicos Locales, los «N eighbourhood $M$ anagement Pathfinders) (la Gestión de Barrios Pioneros), etc.

- Lugares para las personas: asuntos con más base física - planificación urbana, mejora del diseño urbano, me jora de los centros de las ciudades (posibles políticas incluyendo BIDS, inspiradas en los EEUU y en las «H ome Zones) (Zonas de H ogar) de H olanda- .

- Crear y compartir prosperidad: medidas de reactivación económica, incluyendo a las compañías de regeneración urbana (Urban Regeneration Companies), y el Fondo de las Ciudades Inglesas (English Cities Fund). También las estrategias para el crecimiento de la ciudad (City G rowth Strategies), programa piloto basado en el programa de los EEUU para conseguir mayor inversión empresarial en las comunidades en declive. Se han lanzado el crédito impositivo (tax credit) y otras ideas fiscales y financieras.

- Servicios de calidad: mejorar los servicios urbanos clave. Por ejemplo: la salud, las oportunidades para los niños (Sure Start, etc.), además del transporte e iniciativas en la educación (Zonas de Acción Educativa, Excelencia en las ciudades); «Local Public Service Agreements» (cartas de servicios, acuerdos de servicios), donde los Consejos proponen los más altos objetivos;' las iniciativas en materia de vivienda así como los «choice - based lettings pilots» (en relación con los alquileres).

- M edidas fiscales: «rebrownfield» y actividades de renovación.

- Trabajando con las villas y ciudades: partenariados con villas y ciudades.

La agenda más amplia incluye el gran compromiso del Gobierno para prestar un servicio público mejorado e iniciativas tales como la revisión transversal por el Tesoro en la mejora del espacio público. A pesar de esta, a veces mareante, plétora de iniciativas, existe al mismo tiempo una fuerte impresión de que la agenda urbana no es políticamente fuerte. No hay un defensor de las ciudades en el Reino U nido. Específicamente:

- Las ciudades como actores de su propio destino y su futuro parecen débiles. Esto es parcialmente una conse- 
cuencia del centralismo persistente del sistema británico, aunque no puede atribuirse completamente a éste. Con algunas excepciones clave, el liderazgo de la ciudad está pobremente provisto. Blair defiende la elección directa de los Alcaldes como un medio para modernizar el $\mathrm{Go}$ bierno y aportar liderazgo. Pero esta medida ha conocido la resistencia, en líneas generales, durante mucho tiempo, del Gobierno Local, sin alternativa real alguna. Es más, el liderazgo en el nivel central también se ha perdido.

- El sistema de política central-local urbana todavía parece muy top-down (concebido como «enfoque descendente»), a pesar de los maquillajes del partenariado. El Gobierno Central genera nuevas iniciativas;' las ciudades (solas 0 a través de partenariados) solicitan recursos.

\section{La reforma metropolitana principal: el caso de Londres}

La única área de gobernanza urbana donde ha habido un cambio estructural significativo bajo el Nuevo Laborismo es Londres. La creación de la Autoridad del Gran Londres es el último capítulo de la historia de la reforma y del cambio metropolitanos. La relación entre el Estado y la ciudad capital ha sido complicada y difícil durante siglos. A causa de la concentración de población, de actividad económica, política y cultural, los gobiernos siempre se han visto afectados por el potencial y la amenaza del autogobierno de Londres. Londres jugó un papel crucial en la victoria «Roundhead» en la Guerra Civil inglesa del siglo XVII: «pero para la ciudad el Parlamento nunca podría haber hecho la guerra» escribió Thomas H OBBES. Tomando un ejemplo más reciente, $\mathrm{M}$ argaret Thatcher dominaba la $\mathrm{C}$ asa de los $\mathrm{C} 0$ munes, haciendo frente a una pequeña oposición efectiva en el Parlamento. Sin embargo, era diariamente acosada por el gigantesco cartel colgado sobre el edificio del Consejo del Gran Londres, frente al Parlamento, sobre el río, difundiendo el coste de sus políticas económicas sobre el desempleo total de Londres. Londres es demasiado grande, en relación con el resto del país, con una octava parte de la población del Reino U nido y una sexta parte de su Producto Interior Bruto, como para que un Gobierno considere sencillo devolver poder a la capital.

A diferencia de otras muchas ciudades, a medida que Londres iba creciendo, los límites de la antigua ciudad de Londres no se expandieron paralelamente. La ciudad de Londres resistió activamente muchos intentos de reforma y de extensión de sus límites. En 1855 ya existía algún tipo de gobernanza metropolitana en Londres, cuando se constituyó el Consejo M etropolitano de 0 bras Públicas. En 1889, se creó el Consejo del Condado de Londres, cuyos términos formaban lo que ahora se conoce como el «centro de Londres». En 1899, se constituyeron 27 distritos metropolitanos y el Consejo de la Ciudad de Westminster.
Junto con la propia ciudad de Londres, éstas formaban el nivel territorial más bajo de autoridades en un sistema que se mantuvo hasta 1965. En 1965 fue suprimido el Consejo de Condado de Londres y se creó el Consejo del Gran Londres, que cubría 8 millones de residentes y casi toda la zona edificada. En el nivel territorial más bajo se crearon 32 distritos relativamente grandes (con una población media de 250.000 habitantes). Las principales competencias del Consejo del Gran Londres eran la planificación estratégica, la vivienda, los servicios contra incendios y las principales vías públicas. Los distritos eran responsables de los servicios sociales, las viviendas, las vías locales, las bibliotecas, las áreas de recreo y los parques (T RAVERS y JO NES, 1997). La educación en el centro de Londres estaba provista por la Inner London Education Authority. En 1986, el Consejo del Gran Londres fue suprimido, creándose el año 2000 la Autoridad del Gran Londres (Greater London Authority).

\section{6-2000: gobernando sin un centro}

D urante casi un siglo, Londres tuvo un nivel muy elevado de gobierno metropolitano - primero el Consejo de Condado de Londres, y después, desde 1965, el Consejo del Gran Londres- . El Gobierno M etropolitano se terminó de forma abrupta, con una espectacular sesión de fuegos artificiales, a ben eficio de los londinenses, el 31 de marzo de 1986. En la supresión del Consejo del Gran Londres tuvo mucho que ver la decisión personal de la Primera M inistra, M argaret Thatcher. Durante los ochenta, bajo el liderazgo de Ken Livingstone, el Consejo del Gran Londres había desarrollado un enfoque de política de «nueva izquierda» y de retórica antigubernamental. D ebido en parte a su mínimo papel en ese periodo en la prestación directa de servicios públicos, el C onsejo del Gran Londres era capaz de acosar al gobierno y de promover una agenda alternativa de la coalición arco-iris. «Como ningún otro dirigente del Consejo del Gran Londres había hecho anteriormente, Livingstone empleó la riqueza del Condado y su localización al lado del Puente de Westminster como una plataforma para desafiar y pinchar al Gobierno... como el desempleo aumentó alarmantemente a principios de los ochenta, seis figuras de desempleados de L ondres se desplegaron en gigantescas imágenes desde la cornisa del edificio de la sede del Condado» (H EBBERT, 1998: 115). En la decisión de suprimir el Consejo del Gran Londres junto con otras seis autoridades metropolitanas tuvo mucho que ver la decisión personal de la Sra. Thatcher. Esta decisión:

"Fue ampliamente considerada como un acto de rencor político. En las elecciones generales (1983) apareció un manifiesto inesperado. Hacía referencia al extraordinario contraste con las circunstancias de creación del Consejo del G ran Londres, los tres años de deliberaciones de la Comisión H erbert, la compilación 
juiciosa de datos y el examen de evidencia, las audiencias públicas y la cuidadosa búsqueda de consenso... Los simpatizantes se preguntaban cómo podía haberse hecho, cuando la tendencia en las ciudades competidoras de L ondres - Nueva York, Tokio, París, Frankfurt- era de fortalecer las competencias y el estatus de los gobiernos de las ciudades... El Consejo del Gran Londres tuvo muchos críticos, pero la abolición absoluta de todo el gobierno local de Londres estaba fuera de lugar en una agenda política normal y en casi todas las otras ciudades europeas habría sido inconstitudional» (H EBBERT, 1998: 115-116).

Después de la supresión, Londres fue gobernada por una combinación de 33 autoridades de los más bajos niveles territoriales ( 32 distritos, además de la City de Londres); por una diversidad de Consejos conjuntos y comités designados; y a través de gobierno directo y administración por el Gobierno Central. El modelo de gobernanza resultante se ha descrito a menudo como desordenado y fragmentado (NEWMAN y THORNLEY, 1997; Th O Rn LEY, 1998). 0 tro crítico lo describía como «un sis tema de dosniveles territoriales y medio, con distritos en la base, ministerios del gobierno en la parte alta, y rutilando difícilmente entre ambos, una constelación de consejos conjuntos y de comités) (JAM ES, 1990: 145).

Sin embargo - y contradiciendo a muchos críticos al tiempo que predecían su caída- el sistema de gobierno sin un centro funcionaba en cierta medida, e incluso generaba algunos beneficios (véase más adelante). Como John H ALL, un miembro clave y un agudo observador de «London Industry», afirmaba «os nue vos ajustes para administrar los servicios de todo Londres por los comités conjuntos y otros métodos funcionaban tolerablemente bien en la mayoría de los casos. A pesar de los inevitables avisos de que L ondres simplemente fracasaría en su funcionamiento desde el 1 de abril de 1986, ese no fue el caso». (H ALL, 1995). En el período posterior a la abolición, se desarrolló cierta mitología en torno al entonces ausente Consejo del $\mathrm{G}$ ran Londres. A su eliminación se achacó una variedad de patologías urbanas, incluyendo el deterioro de la calidad de vida, el empeoramiento de los servicios públicos, el incremento de personas sin techo y de la desigualdad. En realidad, el Consejo del Gran Londres no había dejado funciones de servicio directo en 1986; su parque de viviendas había sido transferido a los distritos en los setenta y a principios de los ochenta, en parte como respuesta a los pobres resultados en la gestión de la vivienda; y el incremento de la desigualdad en Londres era, sin embargo, en realidad, ampliamente debido al impacto de la economía nacional y a los cambios en las políticas, más que a factores específicos asociados a un supuesto status de ciudad global (Buck, 1997).

El sistema era, no obstante, extremadamente complejo. TRAVERS y JONES (1997) afirmaban que «en realidad, el Gobierno de Londres está vinculado a un amplio número de partenariados formales e informales, comités conjuntos y ajustes de funcionamiento en red». SKELCHER y SteWART (1993) estimaban que había 272 órganos designados en Londres, con un gasto bruto en 1992/1993 de más de 6 mil millones de libras esterlinas. No encontraron apenas distancias entre la cifra de 1.975 Consejeros electos en Londres y los 1.675 designados. Sin embargo, Londres no era excepcional a este respecto, sino más bien estaba en la línea del enorme crecimiento del «Estado no elegido» 0 de la «quangocracia» de los años de Thatcher y M ajor. SKELCHER y STEWART estiman que en 1993 había más de 17.000 miembros de órganos designados en el Reino Unido, frente a los aproximadamente 25.000 Consejeros electos.

El gobierno sin centro generó tanto costes como beneficios para Londres. H ubo cuatro desventajas principales. Primero, el sistema fragmentado de gobernanza hacía más difícil la coordinación y se puede considerar que dejó a Londres sin una planificación estratégica. N EW M AN y T HORNLEY (1997) argumentan que la inicial fragmentación después de la eliminación del Consejo del Gran Londres en 1986, con la planificación, el transporte y otras funciones estratégicas distribuidas a través de un conjunto de distritos, quangos y otras agencias, se incrementó por el desarrollo de una serie entera de proyectos y compañías de desarrollo y regeneración locales y subregionales, en respuesta a la política del gobierno central de competitividad, apostando tanto por la política urbana (por ejemplo, el «City Challenge» y el «Single Regeneration Budget») como por los «N ational Lottery funds:. Cada uno de los 32 barrios y la Corporación de la Ciudad produjo su propio Plan U nitario de D esarrollo, sin ningún órgano de planificación estratégica en el nivel territorial superior. Un comité conjunto, el Comité Consultivo de Planificación de Londres, asesoraba al Secretario de Estado en materia de planificación estratégica en Londres, pero no tenía poderes estatutarios.

En segundo lugar, Londres carecía de voz. Sin un Alcalde o un Consejo M etropolitano, no existía un portavoz o una cabeza visible para defender la posición de Londres a nivel nacional 0 internacional. Esta desventaja era sentida por muchos como una incapacidad de Londres para atraer, o incluso poner en marcha una apuesta créble para grandes acontecimientos internacionales tales como los Juegos O límpicos (H EBBERT, 1998). Es más, incluso se percibía que dentro del Estado nacional Londres a menudo carecía de defensa, sin nadie que enfrentase el punto de vista de muchos miembros del Parlamento y grupos de interés de otras partes de G ran Bretaña de que Londres estaba en una posición privilegiada y favorecida que extraía recursos y atención del resto del país. Sin embargo, la ausencia de una voz en Londres no parecía impedir la capacidad de Londres para acceder, por ejemplo, a los Fondos Estructurales Europeos, y el vacío era cubierto de al gún modo por otras organizaciones - particularmente la Corporación de la Ciudad, los D istritos y la 0 ficina del Gobierno para L ondres (véase más adelante)-. 
En tercer lugar, estaba la cuestión del déficit democrático. Para muchos, era sencillamente inaceptable que una gran ciudad como Londres no tuviese representatividad democrática metropolitana. No se trata sólo de una cuestión funcional, de determinar una visión estratégica para Londres, 0 de mejorar la base económica de Londres, sino también una cuestión política de representatividad y de democracia. El documento de discusión del Partido Laborista A Voice for London (Una voz para Londres) afirmaba:

«La gran mayoría de los londinenses siente claramente que quiere una voz para Londres, hablando en su favor tanto en Gran Bretaña como en la Unión Europea. Ellos sienten que Londres está en desventaja con otras ciudades. 0 tros órganos han desarrollado al gunos aspectos de este rol en Londres, pero no son representantes del pueblo y no tienen legitimidad política para hacer esa labor apropiadamentes.

Por último, la fragmentación no vino acompañada de descentralización, sino de centralización. Se designó un gabinete sub-Comité para Londres en 1992, dirigido por el Secretario de Estado para el M edio Ambiente (T Ravers y JONES, 1997). Se nombró un ministro para Londres y un ministro para el Transporte en Londres. Los miembros de muchos órganos, agencias y otros quangos eran nombrados directamente por los ministros del Gobierno. De manera más importante, en 1993, el Gobierno estableció oficinas del Gobierno en las regiones de Inglaterra para llevar a cabo conjuntamente las actividades regionales de los departamentos centrales o ministerios (H ALL, 1995). De forma significativa, los empleados públicos directores de la 0 ficina del Gobierno para Londres eran de más alto rango que los directores de otras oficinas regionales de Inglaterra. Las tareas clave para la 0 ficina del Gobierno para Londres incluían la distribución de los fondos de rehabilitación y gastos de capital en vivienda, planificación estratégica de los usos del suelo y actuar como punto de contacto con los distritos, las organizaciones de voluntarios y el sector privado (TRAVERS y JONES, 1997). En efecto, el Gobierno Británico - virtualmente por accidentecreó el Prefecto del Támesis, de acuerdo con la nomenclatura francesa. Pero, en el fondo, ésta era una prefectura sin un Alcalde electo o sin una reforma de descentralización al estilo Mitterrand para equilibrarlo:

«N adie que lea el práctico D irectorio de Servicios de la Oficina del Gobierno para Londres podría pasar por alto que con una plantilla detrescientas personas se trataba de un mecanismo poderoso de provisión de servicios, con un completo rango de direcciones, unidades estratégicas, divisiones de planificación, programas de financiación y otros mecanismos institucionales suges tivos de poder político» (TRAVERS y JONES, 1997).

Sin embargo, el presupuesto de mil millones de libras esterlinas de la 0 ficina del Gobierno para Londres era tan sólo una fracción del gasto del Gobierno en Londres. M ientras que la superioridad, personalidad y capacidad de la 0 ficina del Gobierno para Londres le otorgó considerable influencia, «no fueposible, ni podría serlo, una autoridad regional efectiva» (T RAVERS y JONES, 1997: 25).

Compensando estas desventajas se pueden identificar al me nos cuatro tipos de beneficios para Londres en el período posterior a 1986. Primero, y paradójicamente, se ha producido lo que $M$ ichael HebBeRT ha denominado como «la extraña muerte del Gran Londres». Más que un incrementado parroquianismo, hubo un incremento en lo que podría definirse como «la conciencia de Londres» en los pasados 13 años. HeBBert (1995) pone de manifiesto que a mediados de los noventa el término «Gran Londres» estaba desapareciendo de los documentos administrativos correspondientes a las metrópolis urbanizadas:

"Gran" sempre presentó connotaciones de anexión y subordinación. Las percepciones territoriales han cambiado. D esde que fueron absorbidos por el Gran Londres, y resintiéndose durante toda la generación posterior, los barrios han Ilegado a ser hoy parte de L ondres propiamente.. . L os medios de comunicación continuamente refuerzan este sentido de identidad común. L os periódicos, los suplementos, incluso las guías de sucesos, la Televisión de Londres Fin de Semana, y las emisoras de radio tienen asegurado que Londres finaliza exactamente dentro de la circunvalación dela M 25».

Las diferencias en las actitudes y en la política entre el Londres del interior y del extrarradio, aun siendo importantes, están comenzando a atenuarse. Los londinenses se han vuelto cada vez más favorables a una autoridad elegida para Londres, y esto era así tanto en los barrios de las afueras como en los del centro de Londres: el apoyo a favor de una autoridad elegida creció del 47 por ciento en 1987 a un 61 por ciento en la periferia de Londres (HebBert, 1995).

En segundo lugar, existía una experiencia en el trabajo conjunto y una gobernanza en red. La falta de un nivel superior de autoridad, combinada con un sistema confuso y fragmentado adoptado desde 1986, significó que los barrios de Londres tenían que hacerse más cooperativos entre sí, e incluso más competitivos en el sentido de tener que innovar y modernizarse con el fin de obtener financiación adicional. Los políticos suburbanos necesariamente tenían que participar y a veces liderar las redes de políticas públicas de todo Londres. Como se defiende en las teorías funcionalistas de la Ciencia Política, tal rutina de colaboración y participación puede generar lealtades más amplias, a través de la extensión de políticas, compensando, de este modo, las tendencias más parroquianas (H EBBERT, 1995, 1998).

En tercer lugar, y más directamente, hubo un incremento del bipartidismo después de 1986. A pesar de que la batalla de la abolición del Consejo del Gran Londres había sido agria, los 


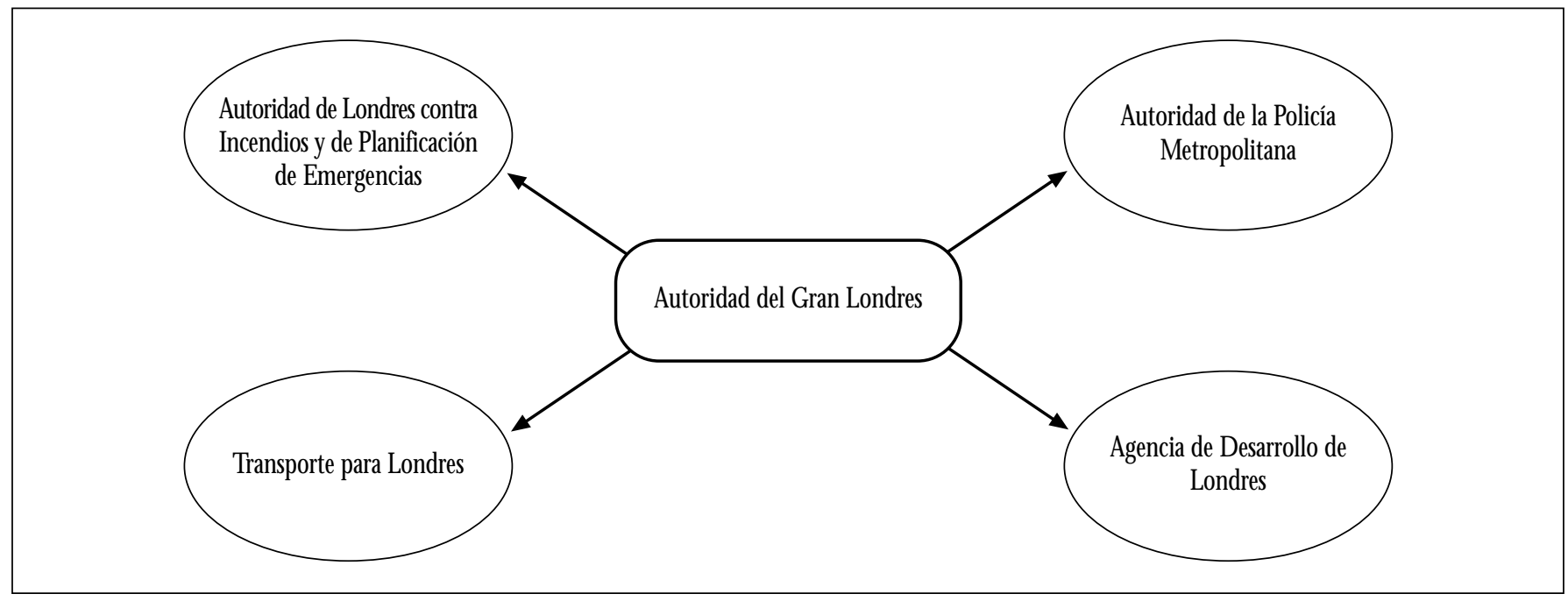

partidos políticos cooperaron sorprendentemente bien en los comités conjuntos y en los consejos, tales como el Comité Consultivo de Planificación de Londres, que se habían puesto en marcha. Entre 1986 y 1994, ningún partido poséa el control absoluto de la mayoría de los barrios de Londres (H ALL, 1995). A pesar de que los barrios se agruparon en dos asociaciones rivales: la Asociación de Barrios Conservadores de Londres y la Asociación de Autoridades Laboristas de Londres, las dos asociaciones fueron capaces de llevar a cabo acciones conjuntas en asuntos tales como la financiación europea, la contaminación medioambiental, la política de tráfico y la de aparcamientos (H EBBERT, 1995). Estas dos asociaciones se fusionaron en los noventa para constituir la Asociación del G obierno de Londres.

Finalmente, el período posterior a 1986 conoció un considerable incremento de la implicación del empresariado en la gobernanza de Londres. En la segunda mitad de los ochenta el lobby de los negocios en Londres se organizó mejor.

En su programa electoral de 1997, el Partido Laborista prometió restablecer el gobierno de toda la ciudad de Londres con un Alcalde elegido directamente y una A samblea. D espués de ese año, el gobierno fijó un documento de consulta, N ew Leadership for London (N uevo Liderazgo para Londres). Fue seguido del Libro Blanco 'A M ayor and Assembly for London' (un Alcalde y una Asamblea para Londres), en marzo de 1998. El 7 de mayo de 1998, los londinenses votaron en referéndum las propuestas del Gobierno. Votaron a favor por un margen del 72 frente al 28 por ciento y una baja participación (35 por ciento). Analizando de barrio a barrio, ningún barrio votó en contra de la propuesta, aunque el apoyo fue generalmente más alto en el centro que en la periferia.

La Greater London Authority Act (Ley de la Autoridad del Gran Londres) es la ley más larga del Parlamento desde la Ley de la India de 1935. Contiene 425 artículos y 34 anexos. N ece sariamente, en esta sección sólo se cita un sumario de los puntos clave.

La Autoridad del Gran Londres se compone de un Alcalde por elección directa y una Asamblea elegida de forma separada, cada uno por un período de cuatro años. La Autoridad del Gran Londres representa una nueva forma de gobernanza en Gran Bretaña, con una clara separación de poderes entre el Alcalde elegido directamente y una pequeña Asamblea de Londres de 25 miembros. El Alcalde es el encargado de desarrollar las estrategias de transporte, planificación, medio ambiente (calidad del aire, contaminación acústica, tratamiento de residuos y bio-diversidad), desarrollo económico y cultural del Gran Londres. El Alcalde es el responsable a la hora de fijar un presupuesto para la Autoridad del Gran Londres y para sus cuatro órganos funcionales: Transporte para Londres (TfL); la Agencia de D esarrollo de Londres (LDA); la Autoridad de Londres contra Incendios y de Planificación de Emergencias (LFEPA); y la Autoridad de la Policía M etropolitana (M PA).

La Asamblea de Londres ha de ser consultada por el Alcalde durante la preparación de cada una de las estrategias de la Autoridad del Gran Londres. La Asamblea considerará el presupuesto para la autoridad y para cada uno de los cuatro órganos funcionales, y puede oponerse al Alcalde con una mayoría de dos tercios. La Asamblea también examinará el ejercicio de las funciones del Alcalde y promoverá investigaciones dentro de los asuntos relacionados con Londres.

El Transporte para Londres está presidido por el Alcalde y posee un amplio conjunto de responsabilidades ejecutivas. La Agencia de D esarrollo de Londres es la encargada del desarrollo económico y la regeneración, y promueve la competitividad y el empleo en Londres. El Alcalde es responsable de la estrategia de desarrollo económico de Londres y ejerce la mayoría de las funciones ejercidas por el Secretario de Estado en relación con las 


\begin{tabular}{|c|c|c|c|c|c|c|}
\hline \multicolumn{7}{|c|}{ Tabla 1} \\
\hline Nombre & Partido & 1a Pref. & $\%$ & 2a Pref.* & $\%$ & Final** \\
\hline Ken Livingstone & Independiente & 667.877 & 39,0 & 178.809 & 12,6 & 776.427 \\
\hline Steve N orris & Conservador & 464.434 & 27,1 & 188.041 & 13,2 & 564.137 \\
\hline Frank Dobson & Laborista & 223.884 & 13,1 & 228.095 & 16,0 & \\
\hline Susan Kramer & Demócrata Liberal & 203.452 & 11,9 & 404.815 & 28,5 & \\
\hline Ram Gidoomal & Alianza de los Pueblos C ristianos & 42.060 & 2,4 & 56.489 & 4,0 & \\
\hline Darren Johnson & Verdes & 38.121 & 2,2 & 192.764 & 13,6 & \\
\hline M ichael N ewland & Partido N acional Británico & 33.569 & 2,0 & 45.337 & 3,2 & \\
\hline Damian Hockney & Partido por la Independencia del Reino Unido & 16.234 & 1,0 & 43.672 & 3,1 & \\
\hline Geoffrey Ben-Nathan & Pro-Pequeñas tiendas de conductore** & 9.956 & 0,6 & 23.021 & 1,6 & \\
\hline Ashwin Kumar Tanna & Independientes & 9.015 & 0,5 & 41.766 & 2,9 & \\
\hline Geoffrey Clements & Partido de la Ley N atural & 5.470 & 0,3 & 18.185 & 1,3 & \\
\hline
\end{tabular}

* Nota del traductor: aśi aparece en el original (Pro-M otorist Small Shop).

Agencias Regionales de D esarrollo en otras partes de Inglaterra. El Alcalde designa a los miembros del Consejo de la Agencia de D esarrollo de Londres, la mayoría de los cuales han de poseer una experiencia en el ámbito de los negocios. No más de cuatro delos doce miembros de la Agencia pueden ser miembros electos.

La Autoridad del Gran Londres posee solamente potestades fiscales muy limitadas. No tiene una potestad general para incrementar los ingresos: no puede elevar los impuestos, ni emitir deuda pública. Puede incrementar los ingresos a través de un precepto identificable para las autoridades de interior del área de Londres, pero está sujeta a los mismos potentes controles centralizados sobre la capacidad impositiva y de gasto que se aplica al resto de autoridades locales británicas. La Autoridad del Gran Londres habrá de obtener sus ingresos también a través de las subvenciones del gobierno, precios por los servicios públicos y ventas de bienes de su patrimonio. Tal vez en el futuro la Autoridad del Gran Londres podría incrementar su presupuesto a través de tasas aplicadas a la congestión urbana u otras «tasas ecológicas» relacionadas con el transporte. La realidad es que «la autonomía fiscal tal como la posee la Autoridad del G ran Londresse limitará a decisiones relacionadas con la asignación de sumas de dinero dentro de su presupuesto global de unas funciones a otras. Incluso aquí, sin embargo, la discrecionalidad de la Autoridad del Gran Londres estará fuertemente restringida... Tal como se concibe en la actualidad, la Autoridad del Gran Londres no posee un grado significativo de autonomía presupuestaria» (LovELAND, 1999).
Las elecciones se celebraron el 4 de mayo del 2000, encontrándose la Autoridad ya operativa el 3 de julio del 2000. El Alcalde fue elegido empleando el sistema de voto suplementario. Los votantes podían expresar una primera y una segunda preferencia. D espués de la primera vuelta, todos los candidatos, excepto los dos primeros, fueron eliminados, y sus votos fueron distribuidos entre los dos restantes atendiendo a sus primeras y segundas preferencias. Los resultados se muestran en la Tabla 1.

Ken Livingstone se llevó el 39 por ciento de los votos de primera preferencia frente al 27 por ciento de su rival más cercano, Steve N orris, con el candidato laborista Frank D obson sólo evitando el cuarto lugar por un estrecho margen. Con las segundas preferencias, Livingstone derrotó a N orris por un 58 por ciento a 42 por ciento. La participación sólo llegó a un decepcionante 34 por ciento. El resultado demostró el predictivo poder de la «Ley deH all»:

«Esta ley (que sin duda es formulada separadamente por muchos observadores de la política londinense) establece que: una elección para el Gran Londres siempre dará lugar a una mayoría opuesta al gobierno nacional del momento» (H ALL 2000: 1).

Votando a Ken Livingstone, muchos londinenses podían reconciliar sus dos preferencias de votar en contra del Gobierno Laborista (que tenía una aplastante mayoría en el Parlamento) y al mismo tiempo evitar apoyar a los Conservadores. D esde fe brero del 2000, Livingstone es miembro del Partido Laborista, y 
ha expresado fervientemente su deseo de volver al redil2. En las primeras horas del 6 de mayo, Ken Livingstone reconocía su victoria iniciando su discurso de toma de posesión: «Como iba diciendo antes de ser interrumpido» (H ALL, 2000).

La Asamblea consta de 25 miembros. Catorce fueron elegidos por distritos, empleando el principio de first past the post ( «el primero consigue el puesto»). Los once restantes fueron elegidos sobre la base de todo L ondres empleando la fórmula $H$ ondt para distribuir los escaños mediante un sistema de representación proporcional. Bajo este sistema, los laboristas y los conservadores obtuvieron 9 escaños cada uno, los demócrata liberales 4 y los verdes 3, dando a la Asamblea de Londres, al igual que la Asamblea galesa y el Parlamento escocés, un aspecto multipartidista no británico, incluso europeo.

Los estilos de voto no fueron, tampoco, los tradicionalmente británicos. En las elecciones de la Autoridad del Gran Londres y de los barrios entre 1964 y 1981, el nivel de voto sumado de los dos grandes partidos nunca bajó del 80 por ciento. Incluso en los ochenta, nunca baió del 70 por ciento. En las elecciones a Alcalde, el voto acumulado sólo fue del 40 por ciento - no sorprendiendo, quizás, el factor Livingstone-. Pero incluso en las elecciones a la Asamblea, el voto acumulado de los dos grandes partidos cayó por debajo del 60 por ciento. Son signos, por tanto, de una nueva política.

\section{Conclusiones sobre la nueva gobernanza metropolitana en Londres}

En los ochenta, el Consejo del Gran Londres, si no fue el líder, sí jugó un papel clave en la cultura de enfrentamiento civil entre el Gobierno Local y el Central. La N ueva I zquierda U rbana empleó su control de las autoridades locales de Londres y de otras grandes ciudades para desafiar no sólo la agenda de N ueva D erecha del gobierno de $M$ argaret Thatcher, sino también, en gran medida, toda la agenda política pro-negocios de los años ochenta.

El contraste con la Autoridad del Gran Londres, la criatura del nuevo milenio, no podría ser más grande. La puesta en marcha de la Autoridad del Gran Londres no indica ciertamente la vuelta del «Gran Gobierno». El Consejo del Gran Londres empleaba una plantilla de en torno a 10.000 empleados. El Alcalde y la Asamblea emplean juntos una plantilla base de en torno a 400 empleados (habrá plantilla adicional en las cuatro agencias clave, por supuesto). Sería muy duro encontrar un ejemplo más dramático del cambio del rowing (remar) al steering (dirigir).

La Autoridad del Gran Londres, en particular el Alcalde, tiene mucho de creación «Blairista» 0 del N uevo Laborismo. Es un animal político muy distinto del antiguo Consejo del Gran Lon- dres, es decir, tanto de la burocracia tecnócrata de los sesenta y setenta, como de la insurgencia de la N ueva Izquierda de los ochenta. El Alcalde es poderoso en su relación con la Asamblea, pero relativamente débil desde el punto de vista financiero y, en términos formales, débil en relación con el Gobierno Central. Pero el potencial para el Alcalde reside en impulsar más capacidad en cuanto a la visibilidad, imagen y personalidad. El Alcalde de Londres es una figura nacional e incluso internacional.

La Autoridad del Gran Londres es, también, en gran medida una creación del $N$ uevo Laborismo en cuanto a que es receptivo al sector empresarial. A pesar de ciertas reservas, la comunidad de los negocios está ampliamente detrás de este cambio institucional. Por su parte, el N uevo Laborismo creó un Alcalde y una Asamblea mucho más del gusto de dicho sector empresarial. Lo que no se previó, por supuesto, es que la primera elección a Alcalde traería de nuevo como vencedor al hombre que simbolizaba para el Primer M inistro exactamente el tipo de política municipal de N ueva Izquierda a la que Tony Blair culpaba de los pobres resultados el ectorales nacionales entre los ochenta y principios de los noventa.

¿Q ué ha cambiado y qué ha continuado desde la creación de la Autoridad del Gran Londres?

Primero, el proceso de gobernanza en red y la práctica de los partenariados, la construcción de coaliciones y el trabajo interagencias ha continuado siendo importante desde el 2000. Esta continuidad entre el período de la desaparición y el período actual refleja:

- Una respuesta racional: el Alcalde/ Autoridad del Gran Londres y otras organizaciones se necesitan entre sí (factores funcionales).

- El efecto de las prácticas y los hábitos que proliferaron en el período sin un nivel metropolitano, entre 1986 y el 2000 (factores organizativos e institucionales).

- El impacto del estilo político del Alcalde - que puede ser aplicable para cualquier Alcalde, pero que es especialmente patente en el caso de Ken LIVINGSTONE (factores políticosy personales)-

En segundo lugar, se mantiene un complejo y fragmentado sistema en el que muchas entidades públicas y privadas diferentes han de trabajar conjuntamente. El Gobierno Central mantiene un papel intervencionista y controlador principal. Los barrios de Londres permanecen como actores clave. M uchos de los partenariados y otras formas de trabajo entre agencias que se de sarrollaron en el período 1986-2000 han sobrevivido a la nueva era, en ocasiones bajo una forma modificada.

En tercer lugar, el Alcalde es poderoso en relación con la A samblea, pero relativamente débil financieramente y en su relación con el Gobierno Central. Los poderes clave del Alcalde son 
la persuasión, el liderazgo y la publicidad. No hace mucho (septiembre del 2002) el Alcalde ha demostrado ser altamente adaptable y exitoso en la nueva política de la gobernanza en red y los partenariados. Sin embargo, la prestación directa de servicios y la implementación todavía es débil. El Alcalde tiende a operar a través de un grupo clave de asesores más que al estilo más tradicional del Gobierno Local o de la Función Pública - irónicamente, esto posee similitudes con los aspectos del Nuevo Laborismo bajo Tony Blair-. Este enfoque posee fortalezas y debilidades. Es más flexible, genera nuevas ideas y es menos burocrático - pero a su vez puede generar confusión, duplicaciones y falta de impulso-.

En cuarto lugar, en los dos primeros años de la existencia de la Asamblea, ésta no ha encontrado un papel efectivo. Los miembros de todos los partidos se quejan en privado de su debilidad colectiva y de su incapacidad para actuar corporativamente. Existe un punto de vista extendido entre otros actores del mundo de la gobernanza londinense sobre la inefectividad de la Asamblea de Londres. Su papel no está claro: para muchos políticos ser un evaluador, y nada más, no resulta atractivo. Los políticos quieren hacer cosas.

En quinto lugar, el Gobierno Central continúa siendo muy importante. La mayor parte del dinero gastado por el Alcalde y los distritos procede deW hitehall. El conflicto más claro, recientemente, con el Gobierno Central ha consistido en el mejor modo de financiar la nueva inversión en el M etro. La escala de gasto del gobierno en Londres ha de tenerse presente. El Alcalde emplea 400 personas y gasta 3 mil millones de libras. El Servicio de Salud $\mathrm{N}$ acional emplea a 140.000 personas en Londres y gasta 5 mil millones de libras. La 0 ficina del Gobierno para Londres no ha desaparecido - de hecho, ahora tiene más departamentos y se ha dotado de una plantilla adicional- .

En sexto lugar, los barrios conservan grandes poderes. Son los responsables primarios de la prestación de servicios públicos. El Alcalde diseña estrategias, pero necesita a los distritos para implementarlas. La relación entre los distritos y la Autoridad del Gran Londres es compleja. No se trata exactamente de que la Autoridad del Gran Londres se dedique exclusivamente a labores estratégicas y los distritos se dediquen simplemente a la prestación de servicios. Ambos niveles de gobierno están implicados tanto en la estrategia como en la prestación de servicios.

\section{¿Más reformas?}

Ya existen demandas de nuevas reformas, y sugerencias sobre el hecho de que los Alcaldes de otras grandes ciudades las dirigen mejor. La creación y puesta en marcha de la nueva autoridad ha reforzado espectacularmente al gunas realidades a largo plazo. Éstas incluyen:
- Westminster y W hitehall no están dispuestos a descentralizar competencias extensivas en un gobierno de todo Londres.

- El gobierno de todo Londres y los distritos de los niveles territoriales más bajos - cada uno con sus propias legitimidades- inevitablemente están condenados al enfrentamiento.

- Las afueras de Londres, a pesar de ser parte de un área urbana continua, están a menudo menos identificadas con los asuntos de todo Londres - la parte central del casco urbano central domina la agenda política de la capital- .

- La fragmentación y el liderazgo débil son las características definitorias de la planificación y el gobierno.

Una nueva reforma es inevitable.

En mayor o menor medida, estos puntos habrían sido tan re levantes en 1830, 1890 o 1970 como lo son hoy. Pero la implicación es evidente: la reforma del 2000 no supuso una solución a largo plazo de los problemas del Gobierno de Londres. Lo que se podría haber hecho, sin embargo, es dotarse de un marco dentro del cual podrían haberse llevado a cabo las futuras reformas. Tales reformas - tal como Tony Travers y yo defendemos en nuestro libro Governing London: Power and Politics in a GIobal City ( G obernando Londres: poder y política en una ciudad global») - necesitaría alcanzar al gunos objetivos:

Una descentralización efectiva de poder desde el Gobierno central al nivel de Londres M etropolitano (esto es, la Autoridad del Gran Londres).

Un reequilibrio de poder entre la Autoridad del Gran Londres y los distritos.

$\square$ Reformar la Autoridad del Gran Londres para asegurar al Alcalde más libertad para designar su plantilla y prestar servicios públicos, acompañada de movimientos para reforzar las competencias de la Asamblea para exigir responsabilidad al Alcalde.

- Una reducción de «quangos» centralmente designados y demás maleza3 gubernamental.

$\square$ Una descentralización fiscal y financiera.

$\square$ Competencias más amplias para el Alcalde de Londres.

\section{Conclusiones generales acerca de la gobernanza metropolitana}

Algunos autores afirman que el principio de competitividad territorial implica que la realidad económica de las regiones urbanas funcionales (FURS) requiere un sistema de gobernanza ur- 
bano-regional para ser efectivo. Esto es, las autoridades públicas necesitan tener la capacidad de influir en un territorio administrativo más amplio que una ciudad estrictamente delimitada desde un punto de vista administrativo. En este caso, las políticas de promoción del crecimiento económico se consideran, en efecto, como una forma de provisión de bienes públicos locales, donde existen importantes difusores y, por tanto, estos «bienes» se ofertan de modo más efectivo a escala regional o cuasi-regional. Este hecho tiene claras implicaciones para la gobernanza: se requiere liderazgo, que puede adoptar la forma de una autoridad regional dominante, un «club» de autoridades, 0 un partenariado con ajustes entre diferentes gobiernos. La teoría también implica que cuanto más unidades existen, más débil es el liderazgo sobre el territorio y más altos los costes de transacción y, por lo tanto, menos efectivas las políticas públicas.

El debate está muy vinculado al concepto de competitividad urbana y, en particular, a la búsqueda de una ventaja competitiva en un delicado proceso de competitividad interurbana. La fragmentación y el parroquianismo se perciben como el proble ma, y el desarrollo de nuevas formas de gobernanza metropolitana - o de ciudad o regional- se conciben como la solución. Como afirma SWANST ROM (2001):

"Lo que defendemos cuando discutimos sobre el regi onalismo son primeramente los costes económi cos y los ben eficios de políticas e instituciones alternativas para el gobierno de las regiones.... Una piedra angular de la nueva literatura regionalista es que las reformas regi onales mejorarán la competitividad de las regiones en la economía global» (p. 480).

Sin embargo, no hay en general una evidencia de que esto sea aś. Por ejemplo, Carr y FeIock (1999) no encontraron certeza en que la consolidación de unidades urbanas más pequeñas en el gobierno metropolitano de nueve ciudades de losEE.UU. tuviese impactos sobre el desarrollo económico. SWAN STROM (2001), en un análisis, encuentra poca certidumbre de que la gobernanza regional fragmentada dificulte el crecimiento económico - « exis te una relación, etá pobremente conectada»—-(p. 482).

El gobierno metropolitano no sólo existe con fines de competitividad - las cuestiones de la cohesión social y la identidad política también son importantes- . Los asuntos públicos de las grandes ciudades de Gran Bretaña son parecidos a los de otras en muchos países europeos. Pero en otros lugares de Europa existen más niveles de gobierno - no es inusual que existan cuatro o incluso cinco niveles- . Así, en París, existen los arrondissements, la Ciudad de París, la Región lle-de- France, y el Estado; mientras que en Roma: Ios municipii; la ciudad de Roma, la Provincia de Roma, la Región del Lazio y el Estado. Por supuesto, en los diferentes países, los distintos niveles de gobierno poseen poderes variados y se producen periódicos intentos de reforma y/o simplificación del sistema. Pero parece ser de mayor aceptación fuera de Gran Bretaña que existan múltiples y solapadas jurisdicciones y responsabilidades y conflictos entre los distintos niveles. En Gran Bretaña, por el contrario, hay continuos temores - particularmente por parte del Gobierno Central- acerca de las consecuencias de más gobiernos y más potencial de conflicto. Este hecho subraya las muy limitadas potestades fiscales y de otra naturaleza garantizadas en la descentralización de Escocia, G ales y Londres.

La forma de la futura gobernanza metropolitana y regional en Gran Bretaña no está todavía clara. Lo que es evidente es la creación de la Autoridad del Gran Londres, con su Alcalde directamente elegido, que ofrecerá un importante y fascinante estudio de caso, relevante no sólo para Gran Bretaña, sino para la gobernanza metropolitana y las políticas públicas en Europa.

Notas

* Ha sido profesor del London School of Economics y de la Universidad de Bristol. En la actualidad es Director del D epartamento de Vivienda del Greater London Authority (GLA).

Traducción española de la versión original inglesa realizada por Pedro Luis Ló PEZ Guerrero, Profesor del Área de Ciencia Política y de la Administración de la Universidad de Alicante.

$1 \mathrm{~N}$ ota del traductor: se traduce el término devolution por devolución, enten- dido como el proceso de descentralización iniciado en Gran Bretaña, a finales de los noventa. Se opta por esta expresión en vez de descentralización, por la propia especificidad de dicho proceso británico al que tal concepto hace referencia.

2 Nota del traductor: con esa misma expresión en el original (expressed his wish to re turn to the fold).

$3 \mathrm{~N}$ ota del traductor: traducción en el sentido del texto governmental undergrowth.

Bibliografía

Buck, N . (1997). «Social Divisions and Labour M arket Change in London; N ational, U rban and G lobal Factors», Working Papers of the ESRC Research Centre on Micro-social Change, Universidad de Essex. Paper n- 97-25.

Buck, N .; Gordon , I.; H all, P.; H ARlOe, M. y Kleinm an, M . (2002). Working Capital: Lifeand Labour in Contemporary London Routledge.
CARR, J. y FEIOCK, R. «M etropolitan Government and Economic D evelopment,» U rban Affairs Review 35 (Enero): 476-488, 1999.

H ALL, J. (1995) «The new parterre of partnerships in London» paper to the ESRC Conference The deregulated city: London in international per spective Reading, Inglaterra. 
H ALL, J. (2000). «H ow London got M ayor Ken (and viceversa)».

H ARding, A. (1990). «Local Autonomy and U rban Economic Development Policies: The Recent UK Experience in Perspectives. En KING , D. y PIERRE, J. (eds.), Challenges to local government Londres: Sage.

H ARDING, A. (1994). «U rban regimes and growth machines: towards a cross-national research agenda» U rban Affairs Q uarterly 29:3, pp. 356-382.

H EBBERT, M. (1995). «Strange D eath of Greater London» paper to ESRC London research seminar Enero 1995.

H EBBERT, M . (1998). London: M oreby Fortunethan D esign, Chichester: Wiley.

JACOBS (1990). «Business Leadership in Urban Regeneration: Towards a Shared Vision?». En KING, D. y PIERRE, J. (eds.), Challenges to local government Londres: Sage.

JOHN y COLE (1995). «M odels of local decision-making networks in Britain and Frances, Policy and Politics 23 (4) 303-312.

Lovelan D, I. (1999). «The Government of London», The Political Quarterly, pp. 91-100.
N eWm An, P. y T horn LeY, A. (1997). «Fragmentation and Centralisation in the Governance of London: Influencing the U rban Policy and Planning Agenda», U rban Studies 34:7, pp. 967-988

N ORTON, A. (1991) «Western European Local Government in Comparative Perspective». En Bat LeY, R. y Sto KeR, G., Local Government in Europe: Trends and D evelopments, M acmillan: Basingstoke.

SkelCher, C. y SteWART, J. (1993). «The appointed government of London», paper prepared for the Association of London Authorities, N oviembre.

SWAN ST ROM , T. (2001). «W hat we argue about when we argue about regionalism», Journal of U rban Affairs 23:5, pp. 476-496.

THORNLEY, A. (1998). «nstitutional change and London's urban policy agenda» ,Annals of Regional Science 32:1, pp.163-183.

Travers, T. y Jones, G. (1997). The N ew Government of London York: Joseph Rowntree Foundation.

Travers, T. y Klein man, M . (2002, forthcoming). Governing London: Power and Politicsin a Global City, Basingstoke: Palgrave. 